\title{
SYNTHESIS, MOLECULAR DOCKING AND ANTIBACTERIAL EVALUATION OF SOME NOVEL N-4 PIPERIDINYL DERIVATIVES OF SPARFLOXACIN
}

\author{
HEMANTH K SUDHEER KUMAR ${ }^{1 *}$, PARAMESHWAR H $^{2}$
}

${ }^{1}$ Department of Pharmaceutical Chemistry, School of Pharmacy and Medical Sciences, Singhania University, Rajasthan, India. ${ }^{2}$ Department of Pharmacognosy, Mother Teresa of Pharmacy, Osmania University, Hyderabad, Telangana, India.

Email: hemanthsudheer3@gmail.com

Received: 15 May 2018, Revised and Accepted: 27 June 2018

ABSTARCT

Objective: The present study envisage a series of sparfloxacin derivatives were synthesized $\left(Q_{1}-Q_{10}\right)$ with added derivatives such as aminomethyl benzenesulfenyl, methyl (methylamino)benzenesulfenyl, amino methyl benzoyl chloride, nitromethyl benzoyl chloride, dimethyl phenylamino, methoxymethyl phenylamino, dimethyl oxopyrazol, methyl dioxopyrrolidine, methyl oxopyrrolidine, and N-Boc amino methyl methylpyrrolidine through N-Piperzinyl linkage.

Methods: All the newly synthesized compounds were characterized by infrared, ${ }^{1} \mathrm{H}$ nuclear magnetic resonance, mass spectrometry, and elemental analysis technique, screened for docking stimulation to find out binding modes of synthesized derivatives with 3FV5 and 3IMW, and evaluated for in vitro antimicrobial activity.

Results: From this study, it was found that the compound $\mathrm{Q}_{5}$ showed good antibacterial activity against Gram-positive (Staphylococcus aureus) and compound $\mathrm{Q}_{4}$ showed good antibacterial activity against Gram-negative (Escherichia coli) in comparison with standard drugs (ciprofloxacin and sparfloxacin). The zone of inhibition and minimum inhibitory concentrations studies performed to synthesized compounds. The correlation between experimental data (minimum inhibitory concentrations) and docking score suggests that penetration for docking simulation is good to mild in reproducing experimental orientation of these synthesized compounds.

Conclusion: The analogs of sparfloxacin are suggested to be potent inhibitors with sufficient scope for further exploration.

Keywords: N-Piperzinyl derivatives, Ciprofloxacin, Sparfloxacin, DNA gyrase, Topoisomerase-IV, Docking studies.

(C) 2018 The Authors. Published by Innovare Academic Sciences Pvt Ltd. This is an open access article under the CC BY license (http://creativecommons. org/licenses/by/4. 0/) DOI: http://dx.doi.org/10.22159/ajpcr.2018.v11i10.27306

\section{INTRODUCTION}

Quinolones have become a major class of antibacterial agents; they have an attraction because of their extremely potent activity, rapid bactericidal effects, and low incidence of resistance development.The main disadvantage of the quinolones is their limited activity against Grampositive pathogens and methicillin-resistant Staphylococcus aureus [1,2]. In addition, quinolones can cause adverse effects, such as central nervous system effects, phototoxicity, tendonitis, hypoglycemia, and serious cardiac dysrhythmias $[3,4]$. Thus, despite many advances in the fluoroquinolones field, there exists continuous need for novel quinolones with better activity profile, pharmacokinetics, and tolerability, to overcome the limitation of existing drugs.The new generations of fluoroquinolones achieved significant improvement in potency, spectrum and physicochemical properties $[5,6]$. The structure-activity relationship studies revealed that the fluorine atom and the 1-alkyl, 1,4-dihydro-4-oxo-quinolone-3-carboxylic acid skeleton of fluoroquinolones are responsible for potency represented in binding with topoisomerase-II DNA gyrase and topoisomerase IV enzymes [7-9]. Topoisomerase II is a target for a variety of quinolones-based drugs. High activity against the eukaryotic type II enzyme is exhibited by drugs contains aromatic substituents at their C-7 position [10].

Sparfloxacin is a fluoroquinolones antibacterial agent active against Gram-positive and Gram-negative bacteria [11]. It has a controversial safety profile and about $37-45 \%$ bound to protein in the blood $[12,13]$. Its structure and ball-stick three-dimensional model have shown in Figs. 1 and 2, respectively.

Skin penetration of sparfloxacin is good. The skin/Plasma ratio was about 1.00 at $4 \mathrm{~h}$ (time of peak plasma concentration) and 1.39 at $5 \mathrm{~h}$ [14]. The compound is used for treating communityacquired lower respiratory tract infection (acute sinusitis, exacerbations of chronic bronchitis caused by susceptible bacteria, and communityacquired pneumonia) [15-17].

The present study reports on the synthesis, spectroscopic analysis including IR and ${ }^{1} \mathrm{H}$ NMR, mass spectrometry and their biological activities of $\mathrm{N}$-piperazinyl derivatives of sparfloxacin $\left(Q_{1}-Q_{10}\right)$.

Molecular docking plays an important role in the rational design of drugs. In the field of molecular modeling, docking is a method which predicts the preferred orientation of one molecule to a second when bound to each other to form a stable complex. Molecular docking can be defined as an optimization problem, which would describe the "best-fit" orientation of a ligand that binds to a particular protein of interest $[18,19]$. The main aim is to evaluate the possible relationship between docking activity of the synthesized compounds $\left(Q_{1}-Q_{10}\right)$ along with interaction with the crystal structure of DNA gyrase of $S$. aureus [PDB:5IWM] and topoisomerase-IV of Escherichia coli [PDB ID: 3FV5].

\section{METHODS}

\section{Experimental}

Procedure for synthesis of (compoundQ $)_{1}$ ) 7-(4-(4-amino-3-methyl benzenesulfenyl)-3,5-dimethylpiperazin-1-yl)-5-amino-1-cyclopropyl6,8-difluoro-1,4-dihydro-4-oxoquinoline-3-carboxylic acid, (compound $\mathrm{Q}_{2}$ ) 7-(4-(3-methyl-4-(methylamino) benzenesulfenyl)3,5-dimethylpiperazin-1-yl)-5-amino-1-cyclopropyl-6,8-difluoro1,4-dihydro-4-oxoquinoline-3-carboxylic acid, (compound $\mathrm{Q}_{7}$ ) 5 -amino-1-cyclopropyl-6,8-difluoro-1,4-dihydro-7-(4-(4,5- 


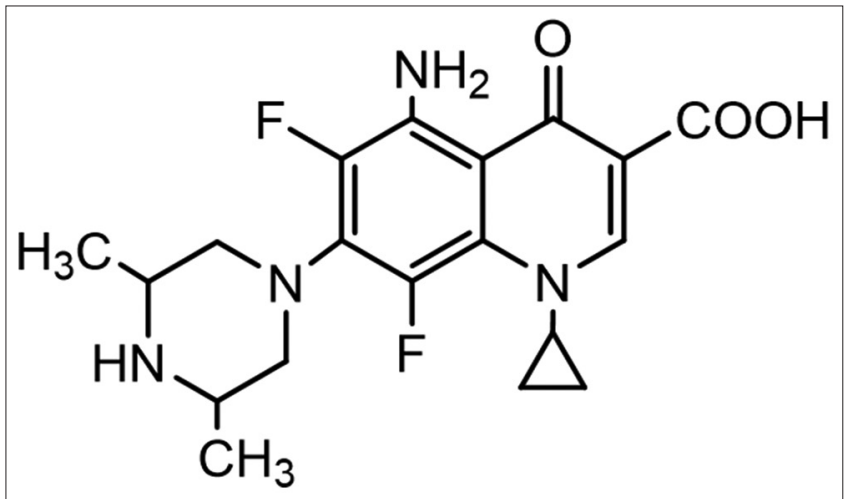

Fig. 1: Sparfloxacin [5-amino-1-cyclopropyl-6,8-difluoro-1,4dihydro-7-(3,5-dimethyl piperazin-1-yi)-4-oxoquinoline-3carboxylic acid]

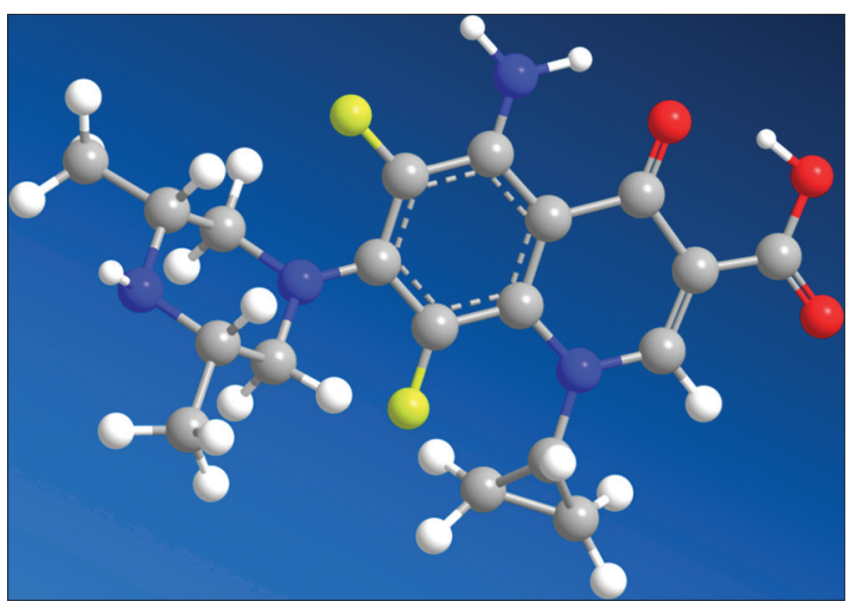

Fig. 2: Sparfloxacin (ball and stick three-dimensional model)

dihydro-3,4-dimethyl-5-oxopyrazol-1-yl)-3,5-dimethylpiperazin1-yl)-4-oxoquinoline-3-carboxylic acid, (compound $\mathrm{Q}_{8}$ ) 5-amino-1-cyclopropyl-6,8-difluoro-1,4-dihydro-7-(3,5-dimethyl-4(3-methyl-2,5-dioxopyrrolidin-1-yl)piperazin-1-yl)-4-oxoquinoline-3carboxylic acid, (compound $Q_{9}$ ) 5-amino-1-cyclopropyl-6,8-difluoro1,4-dihydro-7-(3,5-dimethyl-4-(3-methyl-2-oxopyrrolidin-1-yl) piperazin-1-yl)-4-oxoquinoline-3-carboxylic acid and (compound $\mathrm{Q}_{10}$ ) 5-amino-7-(4-(3-(aminomethyl)-4-methylpyrrolidin-1-yl)3,5-dimethylpiperazin-1-yl)-1-cyclopropyl-6,8-difluoro-1,4dihydro-4-oxoquinoline-3-carboxylic acid from Sparfloxacin (5-amino-1-cyclopropyl-6,8-difluoro-1,4-dihydro-7-(3,5-dimethyl piperazin-1-yi)-4-oxoquinoline-3-carboxylic acid):

To an equimolar amino methyl benzenesulfenyl (derivative $Q_{1}$ ) methyl (methylamino)benzenesulfenyl (derivative of $Q_{2}$ ), dimethyl oxopyrazol (derivative of $\mathrm{Q}_{7}$ ), methyl dioxopyrrolidine (derivative of $\mathrm{Q}_{8}$ ), methyl oxopyrrolidine (derivative of $\mathrm{Q}_{9}$ ), N-Boc amino methyl methylpyrrolidine (derivative of $Q_{10}$ ), and sparfloxacin were added to ethanol, required quantity of formaline (37\%) was added, and the reaction mixture was heated at reflux overnight, cooled. The resulting precipitated solid was filtered and recrystallized from 95\% ethanol $(100 \mathrm{ml})$ to give the product.

Procedure for synthesis of (compound $Q_{3}$ ) 7-(4-(4-amino-3methylbenzoyl)-3,5-dimethylpiperazin-1-yl)-5-amino-1-cyclopropyl-6,8difluoro-1,4-dihydro-4-oxoquinoline-3-carboxylic acid and (compound $\mathrm{Q}_{4}$ ) 7-(4-(4-nitro-3-methylbenzoyl)-3,5-dimethylpiperazin-1-yl)-5-amino1-cyclopropyl-6,8-difluoro-1,4-dihydro-4-oxoquinoline-3-carboxylic acid from Sparfloxacin (5-amino-1-cyclopropyl-6,8-difluoro-1,4-dihydro-7(3,5-dimethyl piperazin-1-yi)-4-oxoquinoline-3-carboxylic acid):
To an equimolar mixture of finely powdered amino methyl benzoyl chloride (derivative of $\mathrm{Q}_{3}$ ), nitromethyl benzoyl chloride (derivative of $Q_{4}$ ) and sparfloxacin were dissolved in $5 \% \mathrm{NaOH}$ solution mix vigorously. The reaction mixture was warmed for an hour and allowed to cool for crystallization. The precipitate was filtered off, washed and dried under vacuum in a desiccator. Sparfloxacin reacts with derivatives in the presence of THF and TEA.

Procedure for synthesis of (compound $Q_{5}$ ) 5-amino-1-cyclopropyl7-(4-((3,4-dimethylphenyl) amino)-3,5-dimethylpiperazin-1yl)-6,8-difluoro-4-oxo-1,4-dihydroquinoline-3-carboxylic acid, (compound $Q_{6}$ ) 5-amino-1-cyclopropyl-6,8-difluoro-7-(4-((4-methoxy3-methylphenyl) amino)-3,5-dimethylpiperazin-1-yl)-4-oxo-1,4dihydroquinoline-3-carboxylic acid from Sparfloxacin (5-amino1-cyclopropyl-6,8-difluoro-1,4-dihydro-7-(3,5-dimethyl piperazin-1-yi)-4-oxoquinoline-3-carboxylic acid):

To an equimolar mixture of sparfloxacin and sodium bicarbonate in $10 \mathrm{ml}$ of acetonitrile was stirred at $50^{\circ} \mathrm{C}$ for $4 \mathrm{~h}$ reaction mixture was cooled to $0^{\circ} \mathrm{C}$ and dimethyl phenyl amino (derivative of $Q_{5}$ ) and methoxy methyl phenyl amino (derivative of $Q_{6}$ ) were added. The mixture was stirred at magnetic stirrer for $5 \mathrm{~h}$ at $0-5^{\circ} \mathrm{C}$ acetonitrile was removed, the precipitate was dried by sodium sulfate, recrystallized in hexane acetone mixture.

\section{Spectral data}

$Q_{1}$ :7-(4-(4-amino-3-methyl benzenesulfenyl)-3,5-dimethylpiperazin1-yl)-5-amino-1-cyclopropyl-6,8-difluoro-1,4-dihydro-4oxoquinoline-3-carboxylic acid

IR $\mathrm{V}_{\max }\left(\mathrm{cm}^{-1} \mathrm{ATR}\right): 3530.6(\mathrm{~N}-\mathrm{H}), 3051.18$ (CH Ar.), 3034.27 (CH cyclopropane), 2619.52 (O-H carboxyl), 1704.80 (C=0 carboxyl), 1622.98 (C=C), 1383 (SO ${ }_{2}$-Piperazine), 1342.61 (C-F), 1270.93 (C-N). ${ }^{1} \mathrm{H}$ NMR (300 MHz; DMSO-d ) $_{6}$ 8:1.075-1.33 (m,4H, Cyclopropane), 1.11 (m,6H, methyl), 2.12(s,3H,methyl), 2.68(m,2H,methine), 3.2-3.5(q, 4H, methylene), $4.12(\mathrm{~m}, 1 \mathrm{H}$, cyclopropane), 6.62 (s, $2 \mathrm{H}$, amine), 6.96-7.24 (d, 3H, benzene), 7.51 (s, 2H,amine), 8.01 (s, 1H, ethylene), 15.12 (s, 1H, Carboxylic acid). MS-ESI: m/z 545.19 (M+1), elemental analysis (\%): $\mathrm{C}_{26} \mathrm{H}_{29} \mathrm{~F}_{2} \mathrm{~N}_{5} \mathrm{O}_{4} \mathrm{~S}$ : C, 57.24; H, 5.36; F, 6.96; N, 12.84; 0, 11.73; S, 5.88.

\section{$Q_{2}$ : 7-(4-(3-methyl-4-(methylamino)benzenesulfenyl)-3,5-}

dimethylpiperazin-1-yl)-5-amino-1-cyclopropyl-6,8-difluoro-1,4dihydro-4-oxoquinoline-3-carboxylic acid

IR $\mathrm{V}_{\max }$ (cm-1 ATR): $3302.34(\mathrm{~N}-\mathrm{H}), 2920.20(\mathrm{CH}), 2805$ (O-H carboxyl), 1442.49 (C=C), 1342.61 ( $\mathrm{SO}_{2}$-Piperazine), 1256.41 (C-N), 1088.94 (C-F). ${ }^{1}$ H NMR (300 MHz; DMSO-d 6 ) $8: 1.075-1.33$ (m,4H, Cyclopropane), 1.11 (m,6H, methyl), 2.12 (s,3H, methyl), 2.68 ( $\mathrm{m}, 2 \mathrm{H}$, methine), 3.2-3.5 (q, 4H, methylene), 4.12 (m, 1H, cyclopropane), 6.62 (s, 2H, amine), 6.96-7.24 (d, 3H, benzene), 7.24 (m, 2H, benzene), 7.51 (s, $2 \mathrm{H}$, amine), 8.01 (s, 1H, ethylene), 15.12 (s, 1H, carboxylic acid). MS-ESI: m/z 555.18 $(\mathrm{M}+1)$, elemental analysis (\%): $\mathrm{C}_{27} \mathrm{H}_{31} \mathrm{~F}_{2} \mathrm{~N}_{5} \mathrm{O}_{4} \mathrm{~S}: \mathrm{C}, 57.24 ; \mathrm{H}, 5.36 ; \mathrm{F}, 6.96$; $\mathrm{N}, 12.84 ; 0,11.73 ; \mathrm{S}, 5.88$.

$Q_{3}:$ 7-(4-(4-amino-3-methylbenzoyl)-3,5-dimethylpiperazin-1-yl)5-amino-1-cyclopropyl-6,8-difluoro-1,4-dihydro-4-oxoquinoline3-carboxylic acid

IR $\mathrm{V}_{\max }$ ( $\mathrm{cm}^{-1} \mathrm{ATR}$ ): 3370.87 (N-H), 3033.93 (CH Ar.), 2978.10 (O-H carboxyl), 2923.14 (C-H cyclopropane), 1703.94 (C=0 carboxyl), 1627.27 $(\mathrm{C}=\mathrm{C}), 1367.51$ ( $\mathrm{SO}_{2}$-Piperazine), $1288(\mathrm{~N}-\mathrm{H}$ sec amine), 1265.08 (CN), $1088.93(\mathrm{C}-\mathrm{F}) .{ }^{1} \mathrm{H}$ NMR (300 MHz; DMSO-d $)$ 8:1.075-1.33 (m,4H, Cyclopropane), 1.31 (m,6H, methyl), 2.12 (s,3H, methyl), 3.465-3.71 (m,4H, methylene), 3.65 (m, 2H, methine), 4.12 (m, 1H, cyclopropane), $6.62(\mathrm{~s}, 2 \mathrm{H}, \mathrm{amine}), 6.93(\mathrm{~d}, 1 \mathrm{H}$, benzene),7.44 (d, 1H, benzene), 7.51 (s, 2H, amine), $7.66(\mathrm{~s}, 1 \mathrm{H}$, benzene), 8.66 (s, 1H, ethylene), $14.93(\mathrm{~s}, 1 \mathrm{H}$, carboxylic acid). MS-ESI: $\mathrm{m} / \mathrm{z} 525.22(\mathrm{M}+1)$, elemental analysis (\%): $\mathrm{C}_{27} \mathrm{H}_{29} \mathrm{~F}_{2} \mathrm{~N}_{5} \mathrm{O}_{4}: \mathrm{C}, 61.71 ; \mathrm{H}, 5.56 ; \mathrm{F}, 7.23 ; \mathrm{N}, 13.33 ; 0,12.18$.

$Q_{4}:$ 7-(4-(4-nitro-3-methylbenzoyl)-3,5-dimethylpiperazin-1-yl)5-amino-1-cyclopropyl-6,8-difluoro-1,4-dihydro-4-oxoquinoline- 


\section{3-carboxylic acid}

IR $\mathrm{V}_{\text {max }}\left(\mathrm{cm}^{-1} \mathrm{ATR}\right): 3069.64$ (CH Ar.), 2871.71 (CH cyclopropane), 2725.33 (O-H carboxyl), 1679.00 (C=0 carboxyl), 1416.37 (C=C), 1322.22 (C-F), 1272.36 (C-N). ${ }^{1} \mathrm{H}$ NMR (300 MHz; DMSO-d $) \delta: 1.075-$ 1.33 (m,4H, Cyclopropane), 1.31 (m,6H, methyl), 2.64 (s,3H, methyl), 3.465-3.71 (m,4H, methylene), $3.65(\mathrm{~m}, 2 \mathrm{H}$, methine), $4.12(\mathrm{~m}, 1 \mathrm{H}$, cyclopropane), 6.62(s, $2 \mathrm{H}$, amine), $8.01(\mathrm{~d}, 1 \mathrm{H}$, benzene), 8.07 (s, $1 \mathrm{H}$, benzene), 8.30 (d, $1 \mathrm{H}$, benzene), $8.66(\mathrm{~s}, 1 \mathrm{H}$, ethylene), 14.93 (s, $1 \mathrm{H}$, carboxylic acid). MS-ESI: $\mathrm{m} / \mathrm{z} 559.19$ (M+1), elemental analysis(\%): $\mathrm{C}_{27} \mathrm{H}_{27} \mathrm{~F}_{2} \mathrm{~N}_{5} \mathrm{O}_{6:} \mathrm{C}, 58.38 ; \mathrm{H}, 4.90 ; \mathrm{F}, 6.84 ; \mathrm{N}, 12.61 ; 0,17.28$.

$Q_{5}:$ 5-amino-1-cyclopropyl-7-(4-((3,4-dimethylphenyl) amino)3,5-dimethylpiperazin-1-yl)-6,8-difluoro-4-oxo-1,4dihydroquinoline-3-carboxylic acid

IR $\mathrm{V}_{\max }$ ( $\mathrm{cm}^{-1}$ ATR): $3370.32(\mathrm{~N}-\mathrm{H}), 3095.32$ (CH Ar.), 2916.52 (C-H cyclopropane), 2652.54 ( $\mathrm{O}-\mathrm{H}$ carboxyl), $1665.33(\mathrm{C}=\mathrm{C}), 1628.25$ ( $\mathrm{C}=\mathrm{O}$ carboxyl), 1332.30 (C-F), 1271.09 (C-N). ${ }^{1}$ H NMR (300 MHz; DMSO-d $)$ $\delta: 1.075-1.33(\mathrm{~m}, 4 \mathrm{H}$, Cyclopropane), 1.27 (m,6H, methyl), 2.19-2.21 (d,6H, methyl), 2.88 (m,2H, methine), 3.285-3.54 (q, 4H, methylene), $4.12(\mathrm{~m}, 1 \mathrm{H}$, cyclopropane), $6.62(\mathrm{~s}, 2 \mathrm{H}$, amine), 6.75-691 (d, $2 \mathrm{H}$, benzene), $6.92(\mathrm{~d}, 1 \mathrm{H}$, benzene), $8.66(\mathrm{~s}, 1 \mathrm{H}$, ethylene), $8.78(\mathrm{~s}, 1 \mathrm{H}$, sec amine), 14.93 (s, 1H, carboxylic acid). MS-ESI: m/z $511.24(\mathrm{M}+1)$, elemental analysis (\%): $\mathrm{C}_{27} \mathrm{H}_{31} \mathrm{~F}_{2} \mathrm{~N}_{5} \mathrm{O}_{3}$ : $\mathrm{C}, 63.69 ; \mathrm{H}, 6.11 ; \mathrm{F}, 7.43 ; \mathrm{N}, 13.69$; $0,9.38$.

$Q_{6}:$ 5-amino-1-cyclopropyl-6,8-difluoro-7-(4-((4-methoxy-3methylphenyl)amino)-3,5-dimethylpiperazin-1-yl)-4-oxo-1,4dihydroquinoline-3-carboxylic acid

IR $\mathrm{V}_{\max }$ ( $\mathrm{cm}^{-1} \mathrm{ATR}$ ): 3069.44 (C-H cyclopropane), 2946.43 (CH Ar.), 2871.73(O-H carboxyl), 2842.41 (OCH3), 1678.12 (C=0 carboxyl), 1415.77 (C=C), 1272.00 (C-N), 1069.39 (C-F). ${ }^{1} \mathrm{H}$ NMR (300 MHz; DMSO- $\mathrm{d}_{6}$ ) $\delta:$ 1.075-1.33 (m,4H, Cyclopropane), 1.27 (m,6H, methyl), 2.15 (s,3H, methyl), 2.88 ( $\mathrm{m}, 2 \mathrm{H}$, methine), 3.28-3.54 (q, 4H, methylene), $3.72(\mathrm{~s}, 3 \mathrm{H}$, methyl), 4.12 (q, $1 \mathrm{H}$, cyclopropane), 6.62(t, $2 \mathrm{H}$, amine), 6.65-6.80 (d, 2H, benzene), 6.92(s, 1H, benzene), 8.66 (s, 1H, ethylene), $8.78(\mathrm{~s}, 1 \mathrm{H}$, sec amine), $14.93(\mathrm{~s}, 1 \mathrm{H}$, carboxylic acid). MS-ESI: $\mathrm{m} / \mathrm{z}$ $527.23(\mathrm{M}+1)$, elemental analysis (\%): $\mathrm{C}_{27} \mathrm{H}_{31} \mathrm{~F}_{2} \mathrm{~N}_{5} \mathrm{O}_{4}$ : C,61.47; $\mathrm{H}, 5.92$; F, $7.20 ; \mathrm{N}, 13.27 ; 0,12.13$.

$Q_{7}:$ 5-amino-1-cyclopropyl-6,8-difluoro-1,4-dihydro-7-(4-(4,5dihydro-3,4-dimethyl-5-oxopyrazol-1-yl)-3,5-dimethylpiperazin1-yl)-4-oxoquinoline-3-carboxylic acid

IR $\mathrm{V}_{\max }\left(\mathrm{cm}^{-1} \mathrm{ATR}\right): 3311.85(\mathrm{~N}-\mathrm{H}), 3057.23$ (CH Ar), 2933.28 (C-H cyclopropane), 2844.14(0-H carboxyl), $1625.38(\mathrm{C}=0$ carboxyl), 1477.71(C=C), $1256.33(\mathrm{C}-\mathrm{N}), \quad 1089.42(\mathrm{C}-\mathrm{F}) .{ }^{1} \mathrm{H} \quad \mathrm{NMR} \quad(300 \mathrm{MHz}$; DMSO-d $) \delta: 0.70$ (d,3H, methyl), 1.075-1.33 (m,4H, Cyclopropane), 1.27 (m,6H, methyl), 1.94 (s,3H, methyl), $2.5(\mathrm{q}, 1 \mathrm{H}$, methine), $2.88(\mathrm{~m}$, $2 \mathrm{H}$, methine), 3.28-3.54 (q,4H, methylene), 4.12 ( $\mathrm{m}, 1 \mathrm{H}$, cyclopropane), $6.62(\mathrm{~s}, 2 \mathrm{H}, \mathrm{amine}), 8.66(\mathrm{~s}, 1 \mathrm{H}$, ethylene), $14.93(\mathrm{~s}, 1 \mathrm{H}$, carboxylic acid). MS-ESI: $\mathrm{m} / \mathrm{z} 502.41(\mathrm{M}+1)$, elemental analysis (\%): $\mathrm{C}_{24} \mathrm{H}_{28} \mathrm{~F}_{2} \mathrm{~N}_{6} \mathrm{O}_{3}$ : C,57.36; H, 5.62; F, 7.56; N, 16.72; 0, 12.73 .

\section{$Q_{8}:$ 5-amino-1-cyclopropyl-6,8-difluoro-1,4-dihydro-7-(3,5-} dimethyl-4-(3-methyl-2,5-dioxopyrrolidin-1-yl)piperazin-1-yl)-4oxoquinoline-3-carboxylic acid

IR $\mathrm{V}_{\max }\left(\mathrm{cm}^{-1} \mathrm{ATR}\right): 3373.50(\mathrm{~N}-\mathrm{H}), 3084.24$ (CH Ar.), $2915.38(\mathrm{O}-\mathrm{H}$ carboxyl), 1628.46 (C=0 carboxyl), 1470.32 (C=C), 1217.17 (C-N), $1025.73(\mathrm{C}-\mathrm{F}) .{ }^{1} \mathrm{H}$ NMR (300 MHz; DMSO-d ${ }_{6}$ ) $\delta: 1.075-1.33(\mathrm{~m}, 4 \mathrm{H}$, Cyclopropane), 1.17 (d,3H, methyl), 1.27 (m,6H, methyl), 2.61-2.85 ( $\mathrm{m}, 3 \mathrm{H}$, succinimide), 2.88 ( $\mathrm{m}, 2 \mathrm{H}$, methane), 3.28-3.54 (q,4H, methylene), $4.12(\mathrm{~m}, 1 \mathrm{H}$, cyclopropane), 6.62 (s, 2H, amine), 8.66 (s, 1H, ethylene), 14.93 (s, 1H, carboxylic acid). MS-ESI: m/z $505.90(\mathrm{M}+1)$, elemental analysis (\%): $\mathrm{C}_{24} \mathrm{H}_{27} \mathrm{~F}_{2} \mathrm{~N}_{5} \mathrm{O}_{5}$ : C,57.25; H, 5.4; F, 7.55; N, 13.91; O, 15.89 .

$Q_{9}$ : 5-amino-1-cyclopropyl-6,8-difluoro-1,4-dihydro-7-(3,5dimethyl-4-(3-methyl-2-oxopyrrolidin-1-yl)piperazin-1-yl)-4- oxoquinoline-3-carboxylic acid

IR $\mathrm{V}_{\max }$ ( $\mathrm{cm}^{-1} \mathrm{ATR}$ ): 3377.55 (N-H), 3084.48 (CH Ar.), 2849.11 (C-H cyclopropane), 2687.43 (O-H carboxyl), 1703.90 (C=0 carboxyl), 1622.59 (C=C), 1342.49 (C-F), 1270.55 (C-N). ${ }^{1} \mathrm{H}$ NMR (300 MHz; DMSO-d $\left.{ }_{6}\right) \delta: 1.075-1.33$ (m,4H, Cyclopropane), 1.12 (d,3H, methyl), 1.27 (m,6H, methyl), 1.91-2.17 ( $\mathrm{m}, 2 \mathrm{H}$,pyrrolidine), 2.33 (m,1H, pyrrolidine2-one), 2.88 (m, 2H, methine), 3.24-3.34 ( $\mathrm{m}, 2 \mathrm{H}$,pyrrolidine-2-one), 3.28-3.54 (q,4H, methylene), 4.12 (m, 1H, cyclopropane), $6.62(\mathrm{~s}, 2 \mathrm{H}$, amine), $8.66(\mathrm{~s}, 1 \mathrm{H}$, ethylene), $14.93(\mathrm{~s}, 1 \mathrm{H}$, carboxylic acid).MS-ESI: $\mathrm{m} / \mathrm{z} 489.22(\mathrm{M}+1)$, elemental analysis(\%): $\mathrm{C}_{24} \mathrm{H}_{29} \mathrm{~F}_{2} \mathrm{~N}_{5} \mathrm{O}_{4}: \mathrm{C}, 58.59 ; \mathrm{H}$, 5.97; F, 7.76; N, 14.31; 0, 13.07 .

$Q_{10}:$ 5-amino-7-(4-(3-(aminomethyl)-4-methylpyrrolidin-1-yl)3,5-dimethylpiperazin-1-yl)-1-cyclopropyl-6,8-difluoro-1,4dihydro-4-oxoquinoline-3-carboxylic acid

IR $\mathrm{V}_{\max }\left(\mathrm{cm}^{-1} \mathrm{ATR}\right)$ : $3382.18(\mathrm{~N}-\mathrm{H}), 3084.70$ (CH Ar.), 3100 (C-H cyclopropane), 2915.29 (O-H carboxyl), 1660.31 (C=0 carboxyl), 1405.09 (C=C), 1332.37 (C-F), 1271.72 (C-N). ${ }^{1} \mathrm{H}$ NMR (300 MHz; DMSO- $_{6}$ ) $\delta: 0.93$ (d,3H, methyl), 1.075-1.33 (m,4H, Cyclopropane), 1.27 (m,6H, methyl), 1.5 (m,2H,amine), 1.44 (s,1H,pyrrolidine), 1.59 (m,1H,pyrrolidine), 2.44-2.69 (q,2H, methylene), 2.6-2.81 (q,4H,pyrrolidine), 2.88 ( $\mathrm{m}, 2 \mathrm{H}$, methine), 3.28-3.54 (q,4H,methylene), $4.12(\mathrm{~m}, 1 \mathrm{H}$, cyclopropane), $6.62(\mathrm{~s}, 2 \mathrm{H}$, amine), 8.66 (s, 1H, ethylene), 14.93 (s, 1H, carboxylic acid). MS-ESI: m/z $504.27(\mathrm{M}+1)$, elemental analysis(\%): $\mathrm{C}_{25} \mathrm{H}_{34} \mathrm{~F}_{2} \mathrm{~N}_{6} \mathrm{O}_{8}$ : C,59.51; $\mathrm{H}, 6.79 ; \mathrm{F}, 7.53 ; \mathrm{N}, 16.66 ; 0,9.51$.

\section{Biological evaluations}

Antimicrobial activity

All the title compounds were screened for their antibacterial and antifungal activities. The antibacterial activity of the synthesized compounds was tested against two Gram-positive bacteria (S. aureus ATCC 6438P and Staphylococcus epidermidis ATCC 155) and two Gramnegative bacteria (E. coli ATCC 25922 and Klebsiella pneumoniae ATCC 29665) using nutrient agar medium. The antifungal activities of the compounds were tested against two fungi, namely Aspergillus niger ATCC 9029 and Aspergillus fumigatus ATCC 46645 using Sabouraud dextrose agar. For preliminary screening, the antimicrobial tests were carried out by the paper disc diffusion method. The minimum inhibitory concentrations (MIC) of the compounds were also determined by agar streak dilution method.

\section{Paper disc diffusion technique}

The sterilized [20] (autoclaved at $120^{\circ} \mathrm{C}$ for $30 \mathrm{~min}$ ) medium $\left(40-50^{\circ} \mathrm{C}\right.$ ) was inoculated $\left(1 \mathrm{ml} / 100 \mathrm{ml}\right.$ of medium) with the suspension $\left(10^{5}\right.$ $\mathrm{cfu} / \mathrm{ml}$ ) of the microorganism (matched to McFarland barium sulfate standard) and poured into a Petri dish to give a depth of 3-4 $\mathrm{mm}$. The paper impregnated with the test compounds $(100 \mu \mathrm{g} / \mathrm{ml}$ in dimethylformamide) was placed on the solidified medium. The plates were pre-incubated for $1 \mathrm{~h}$ at room temperature and incubated at $37^{\circ} \mathrm{C}$ for 24 and $48 \mathrm{~h}$ for antibacterial and antifungal activities, respectively. Ciprofloxacin $(100 \mu \mathrm{g} / \mathrm{disc})$ and ketoconazole $(100 \mu \mathrm{g} / \mathrm{disc})$ were used as a standard for antibacterial and antifungal activities, respectively. The observed zone of inhibition is presented in Table 1.

\section{MIC}

MIC [21] of the compound was determined by agar streak dilution method. A stock solution of the synthesized compound $(100 \mu \mathrm{g} / \mathrm{ml})$ in dimethylformamide was prepared, and graded quantities of the test compounds were incorporated in a specified quantity of molten sterile agar (nutrient agar for antibacterial activity and Sabouraud dextrose agar medium for antifungal activity). A specified quantity of the medium $\left(40-50^{\circ} \mathrm{C}\right)$ containing the compound was poured into a Petri dish to give a depth of 3-4 $\mathrm{mm}$ and allowed to solidify. Suspension of the microorganism was prepared to contain approximately $10^{5} \mathrm{cfu} / \mathrm{ml}$ and applied to plates with serially diluted compounds in dimethylformamide to be tested and incubated at $37^{\circ} \mathrm{C}$ for $24 \mathrm{~h}$ and 
Table 1: Antimicrobial activity of the synthesized compounds (Q1-Q10) $(100 \mu \mathrm{g} / \mathrm{ml})$

\begin{tabular}{|c|c|c|c|c|c|c|}
\hline \multirow[t]{3}{*}{ Compounds } & \multicolumn{6}{|c|}{ In vitro activity - zone of inhibition in $\mathrm{mm}$ (MIC in $\mu \mathrm{g} / \mathrm{ml}$ ) } \\
\hline & \multicolumn{2}{|c|}{ Gram-positive bacteria } & \multicolumn{2}{|c|}{ Gram-negative bacteria } & \multicolumn{2}{|l|}{ Fungi } \\
\hline & S. aureus & S. epidermidis & E. coli & K. pneumoniae & A. niger & A. fumigatus \\
\hline Q1 & $28(1.2)$ & $25(3.4)$ & $23(1.2)$ & $24(4.2)$ & 18 (14.1) & 23 (15.6) \\
\hline Q2 & $29(1.3)$ & $24(3.3)$ & $26(0.8)$ & $24(2.8)$ & 19 (13.6) & $24(14.9)$ \\
\hline Q3 & $29(1.6)$ & $26(3.8)$ & 26 (1.9) & $22(3.5)$ & $17(13.8)$ & $23(15.2)$ \\
\hline Q4 & $30(1.4)$ & $26(2.6)$ & $30(0.9)$ & $31(2.2)$ & $18(13.9)$ & $20(14.8)$ \\
\hline Q5 & $32(1.1)$ & $31(2.2)$ & $22(2.2)$ & $20(3.9)$ & $23(14.2)$ & 23 (15.6) \\
\hline Q6 & $32(1.2)$ & $28(2.9)$ & $23(1.0)$ & $22(3.8)$ & $21(14.7)$ & $21(16.2)$ \\
\hline Q7 & $24(2.4)$ & $24(3.1)$ & 27 (1.8) & $24(4.1)$ & 25 (13.2) & $25(14.3)$ \\
\hline Q8 & $21(2.0)$ & $25(3.3)$ & $29(1.1)$ & $27(2.3)$ & 25 (12.9) & 24 (13.1) \\
\hline Q9 & $29(1.4)$ & $27(2.5)$ & $28(1.7)$ & $26(3.5)$ & $26(12.7)$ & 27 (13.8) \\
\hline Q10 & $28(2.5)$ & $21(3.1)$ & $28(1.9)$ & $23(2.6)$ & $22(14.1)$ & $21(14.8)$ \\
\hline Sparfloxacina & $34(0.7)$ & $33(0.8)$ & $34(0.2)$ & $33(0.1)$ & - & - \\
\hline Ciprofloxacin ${ }^{\mathrm{a}}$ & $37(0.5)$ & $35(0.12)$ & $36(0.06)$ & $36(0.06)$ & - & - \\
\hline Ketoconazole $^{\mathrm{b}}$ & - & - & - & - & $29(10.8)$ & 33 (11.4) \\
\hline $\mathrm{DMF}^{\mathrm{c}}$ & - & - & - & - & - & - \\
\hline
\end{tabular}

Sparfloxacin ${ }^{\mathrm{a}}$, Ciprofloxacina : Standard antibacterial drugs, Ketoconazole ${ }^{\mathrm{b}}$ : Standard antifungal drug and DMF : Control. S. aureus: Staphylococcus aureus, S. epidermidis: Staphylococcus epidermidis, E. coli: Escherichia coli, K. pneumonia: Klebsiella pneumonia, A. niger: Aspergillus niger, A. fumigatus: Aspergillus fumigatus. MIC: Minimum inhibitory concentrations

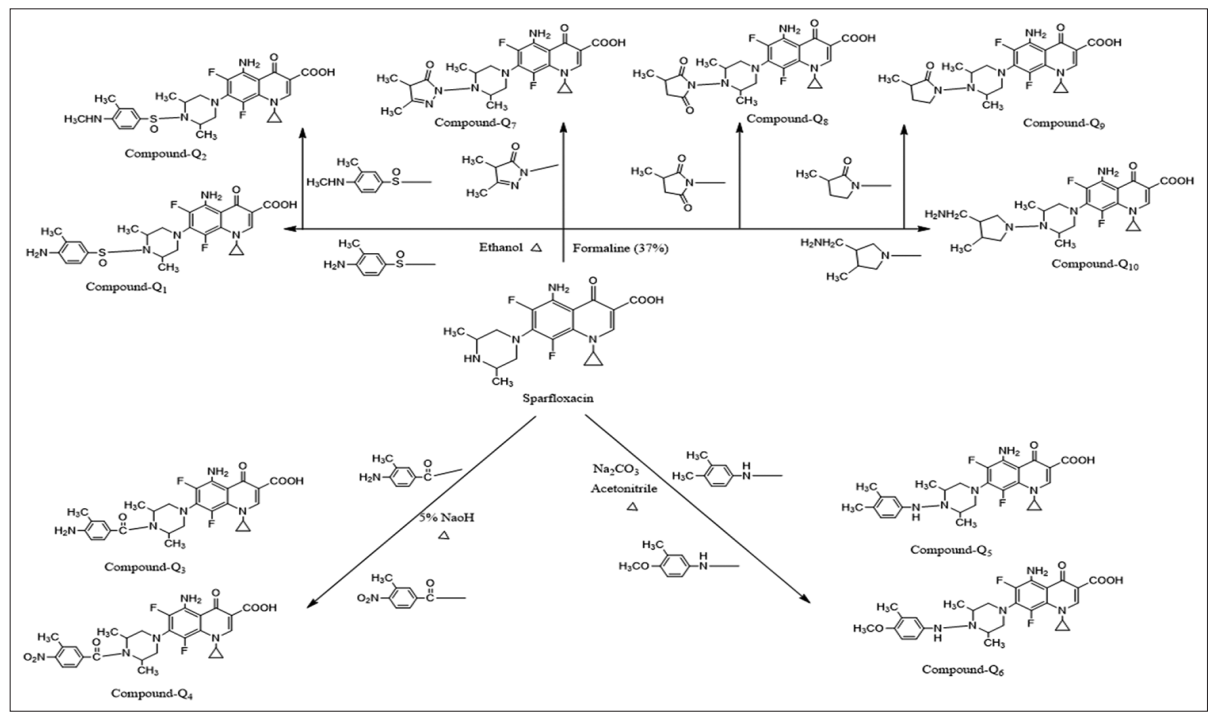

Scheme 1: Synthesis of N-Piperzinyl derivatives of sparfloxacin

$48 \mathrm{~h}$ for bacteria and fungi, respectively. The MIC was considered to be the lowest concentration of the test substance exhibiting no visible growth of bacteria or fungi on the plate. The observed MIC is presented in Table 1 .

\section{Molecular docking studies of sparfloxacin}

Molecular docking studies of synthesized compounds $Q_{1}-Q_{10}$ with wellestablished structure of $S$. aureus and $E$. coli were performed using Auto Dock vina 1.12 version and chimera 1.12 version. The binding pocket of the active site of DNA gyrase (PDB: 5IWM) for Gram-positive bacteria like S. aureus and (PDB: 3FV5) for Gram-negative bacteria like E. coli. Docking method involves the following steps. First, the ligand molecule was build, in the second step required protein was downloaded from PDB, preparation, and validation of macromolecule by X-ray crystallography. Third step is the identification of binding affinity by the extent of binding of a ligand to the protein of molecule.

\section{RESULTS AND DISCUSSION}

\section{Chemistry}

The synthetic route to obtain the necessary derivative from commercially available reagent is briefly outlined in Scheme 1 . The synthesized compounds $\left(\mathrm{Q}_{1}-\mathrm{Q}_{10}\right)$ were obtained with the help of various derivatives and reagents such as formaline (37\%), $5 \% \mathrm{NaOH}, \mathrm{Na}_{2} \mathrm{CO}_{3}$, and acetonitrile. All reactions of synthesized compounds occurred with optimum temperature. The structure of all synthesized compounds was confirmed by IR, ${ }^{1} \mathrm{HNMR}$, and mass spectral elemental analysis techniques.

\section{Antibacterial activity}

All the synthesized compounds $\left(Q_{1}-Q_{10}\right)$ were tested zone of inhibition and MIC values against two Gram-positive (S. aureus and S. epidermidis) and two Gram-negative (E. coli and K. Pneumonia) bacteria. All the compounds exhibited mild to moderate activity against both Gram-positive and Gramnegative bacteria. Compounds $Q_{5}, Q_{6}, Q_{4}$, and $Q_{3}$ were found to possess significant antibacterial activity against Gram-positive organisms when compared to standard drugs (ciprofloxacin and sparfloxacin). Compounds $Q_{4}, Q_{8^{\prime}}, Q_{9}$, and $Q_{10}$ were found to possess significant antibacterial activity against Gram-negative organisms when compared to standard drugs (ciprofloxacin and sparfloxacin). The synthesized compounds exhibited MIC values in the range of $0.8-4.2 \mu \mathrm{g} / \mathrm{ml}$ shown in Table 1 .

Compound $Q_{5}$ exhibited mild antibacterial activity with MIC value in the range of $(1.12 \mu \mathrm{g} / \mathrm{ml})$ when compared to standard sparfloxacin 
Table 2: Docking result of synthesized compounds $\left(Q_{1}-Q_{10}\right)$ with MIC values $(\mu \mathrm{g} / \mathrm{ml})$

\begin{tabular}{|c|c|c|c|c|c|c|}
\hline \multirow[t]{2}{*}{ Compounds } & \multicolumn{3}{|c|}{ Gram-positive bacteria ( $S$. aureus) } & \multicolumn{3}{|c|}{ Gram-negative bacteria ( $E$. coli ) } \\
\hline & $\begin{array}{l}\text { Docking } \\
\text { Score }^{\mathrm{a}}\end{array}$ & $\mathrm{MIC}(\mu \mathrm{g} / \mathrm{ml})$ & H bonds with 5IWM enzyme & $\begin{array}{l}\text { Docking } \\
\text { Score }^{\mathrm{a}}\end{array}$ & $\operatorname{MIC}(\mu \mathrm{g} / \mathrm{ml})$ & $\begin{array}{l}\text { Interactions with } 3 F V 5 \\
\text { enzyme }\end{array}$ \\
\hline Q1 & -9.2 & 1.2 & $\begin{array}{l}3^{\text {rd }} \text { position } \mathrm{COOH} \text { oxygen with } \\
\text { ALA138 and } 5^{\text {th }} \text { position amino } \\
\text { hydrogen with GLY963 }\end{array}$ & -7.1 & 1.2 & $\begin{array}{l}5^{\text {th }} \text { position amino hydrogen } \\
\text { with ARG79 }\end{array}$ \\
\hline Q2 & -9.2 & 1.3 & $\begin{array}{l}5^{\text {th }} \text { position amino } \\
\text { hydrogen with ASP } 475 \\
\text { and methyl (methylamino) } \\
\text { benzenesulfenyl amino } \\
\text { hydrogen with ALA459 }\end{array}$ & -7.2 & 0.8 & $\begin{array}{l}5^{\text {th }} \text { position amino nitrogen } \\
\text { with ILE81 }\end{array}$ \\
\hline Q3 & -9.4 & 1.6 & $\begin{array}{l}3^{\text {rd }} \text { position COOH Oxygen with } \\
\text { GLY958 and Amino methyl } \\
\text { benzoyl hydrogen with LYS28 }\end{array}$ & -7.0 & 1.9 & $\begin{array}{l}5^{\text {th }} \text { position amino hydrogens } \\
\text { with ARG124 And ASP56 and } \\
\text { nitrogen with THR126 }\end{array}$ \\
\hline Q4 & -9.5 & 1.4 & $\begin{array}{l}3^{\text {rd }} \text { position } \mathrm{COOH} \text { hydrogen } \\
\text { with GLY287, } 5^{\text {th }} \text { position amino } \\
\text { hydrogens with ALA275 and } \\
\text { GLY287 nitromethyl benzoyl } \\
\text { oxygen with ILE312 }\end{array}$ & -7.6 & 0.9 & $\begin{array}{l}\text { Nitromethyl benzoyl oxygen } \\
\text { forms hydrogen bond with } \\
\text { ALA47 }\end{array}$ \\
\hline Q5 & -9.8 & 1.1 & $\begin{array}{l}3^{\text {rd }} \text { position COOH Oxygen with } \\
\text { MET294, } 5^{\text {th }} \text { position amino } \\
\text { hydrogen with GLY292 and } \\
\text { dimethyl phenylamino hydrogen } \\
\text { with GLY287 }\end{array}$ & -7.2 & 2.2 & $\begin{array}{l}5^{\text {th }} \text { position amino hydrogen } \\
\text { with THR126 }\end{array}$ \\
\hline Q6 & -9.6 & 1.2 & $\begin{array}{l}4^{\text {th }} \text { Position Oxygen with } \\
\text { GLY958 and } 5^{\text {th }} \text { position amino } \\
\text { hydrogens with GLU997 and } \\
\text { PR0365 and amino nitrogen } \\
\text { with GLY958 }\end{array}$ & -7.1 & 1.0 & $\begin{array}{l}3^{\text {rd }} \text { position } \mathrm{COOH} \text { hydrogen } \\
\text { with GLY59 }\end{array}$ \\
\hline Q7 & -8.8 & 2.4 & $\begin{array}{l}5^{\text {th }} \text { position amino hydrogen } \\
\text { with GLY958 and Dimethyl } \\
\text { oxopyrazol oxygen with ILE983 }\end{array}$ & -7.2 & 1.8 & $\begin{array}{l}\text { Dimethyl oxopyrazol oxygen } \\
\text { with ILE81 }\end{array}$ \\
\hline Q8 & -8.6 & 2.0 & $\begin{array}{l}3^{\text {rd }} \text { position COOH oxygen with } \\
\text { PHE962 and Methyl dioxo } \\
\text { pyrrolidine Piperazine Oxygen } \\
\text { with ILE1154 }\end{array}$ & -7.4 & 1.1 & $\begin{array}{l}\text { Methyl dioxo pyrrolidine } \\
\text { Piperazine oxygen with ILE81 }\end{array}$ \\
\hline Q9 & -8.6 & 1.4 & $\begin{array}{l}5^{\text {th }} \text { position amino hydrogen } \\
\text { with GLY958 and } 3^{\text {rd }} \text { position } \\
\text { COOH oxygen with ALA138 }\end{array}$ & -7.4 & 1.7 & $\begin{array}{l}\text { Methyl oxo pyrrolidine } \\
\text { Piperazine oxygen with SER } 82\end{array}$ \\
\hline Q10 & -8.2 & 2.5 & $\begin{array}{l}3^{\text {rd }} \text { position COOH oxygen } \\
\text { with SER } 22 \text { and ASP } 21 \\
\text { and Aminomethyl methyl } \\
\text { pyrrolidine hydrogens with } \\
\text { DA1349 and DG1369 }\end{array}$ & -7.3 & 1.9 & $\begin{array}{l}\text { Aminomethyl } \\
\text { methylpyrrolidine hydrogen } \\
\text { with LEU75 }\end{array}$ \\
\hline Sparflo-xacin ${ }^{b}$ & -8.2 & 0.7 & $\begin{array}{l}3^{\text {rd }} \text { position } \mathrm{COOH} \text { oxygen with } \\
\text { ALA970 and } 5^{\text {th }} \text { position amino } \\
\text { hydrogens with ASP968 and } \\
\text { SER964 }\end{array}$ & -7.2 & 0.2 & $\begin{array}{l}3^{\text {rd }} \text { position } \mathrm{COOH} \text { hydrogen } \\
\text { with HIS } 37,5^{\mathrm{TH}} \text { Position amino } \\
\text { nitrogen with THR } 126 \text { and } \\
\text { hydrogen with THR } 126\end{array}$ \\
\hline Ciproflo-xacin ${ }^{b}$ & -7.8 & 0.5 & $\begin{array}{l}7^{\text {th }} \text { position Piperazine hydrogen } \\
\text { with VAL1120 and } 3^{\text {rd }} \text { position } \\
\text { COOH oxygen with ALA790 }\end{array}$ & -7.3 & 0.06 & $\begin{array}{l}7^{\text {th }} \text { position Piperazine } \\
\text { hydrogen with ASP56 }\end{array}$ \\
\hline
\end{tabular}

${ }^{a}$ Based on Auto Dock Vina score, ${ }^{b}$ Sparfloxacin, ciprofloxacin (standard drugs). MIC: Minimum inhibitory concentrations, S. aureus: Staphylococcus aureus,

E.Coli: Escheriachia coli

$(0.7 \mu \mathrm{g} / \mathrm{ml})$ and ciprofloxacin $(0.5 \mu \mathrm{g} / \mathrm{ml})$. This mild antibacterial activity is maybe due to addition of new derivative dimethyl phenylamino group at the $7^{\text {th }}$ position of piperzinyl ring.

Compound $\mathrm{Q}_{4}$ exhibited mild Gram-negative antibacterial with MIC values in the range of $(0.9 \mu \mathrm{g} / \mathrm{ml})$ when compared to standard sparfloxacin $(0.2 \mu \mathrm{g} / \mathrm{ml})$ and ciprofloxacin $(0.06 \mu \mathrm{g} / \mathrm{ml})$. This mild antibacterial activity is maybe due to the addition of new derivative nitromethyl benzoyl group at the $7^{\text {th }}$ position of piperzinyl ring.

\section{Antifungal activity}

All the synthesized compounds $\left(Q_{1}-Q_{10}\right)$ were tested zone of inhibition and MIC values against two fungi organisms (A. niger and A. fumigatus).
All the compounds exhibited mild to moderate activity. Compounds $Q_{g}$, $Q_{8}$, and $Q_{7}$ were found to possess significant antifungal activity against A. niger when compared to standard ketoconazole. Compounds $Q_{9}, Q_{2}$, and $Q_{7}$ were found to possess significant antifungal activity against A. fumigatus when compared to standard drug ketoconazole. The synthesized compounds exhibited MIC values in the range of 12.7$16.2 \mu \mathrm{g} / \mathrm{ml}$ shown in Table 1 .

Compound $\mathrm{Q}_{9}$ exhibited mild antifungal activity with MIC values of $(12.7 \mu \mathrm{g} / \mathrm{ml})$ and $(13.8 \mu \mathrm{g} / \mathrm{ml})$ when compared to standard ketoconazole $(10.8 \mu \mathrm{g} / \mathrm{ml})$ and $(11.4 \mu \mathrm{g} / \mathrm{ml})$. This mild antifungal activity is maybe due to the addition of new derivative methyl oxopyrrolidine group at the $7^{\text {th }}$ position of piperzinyl ring. 


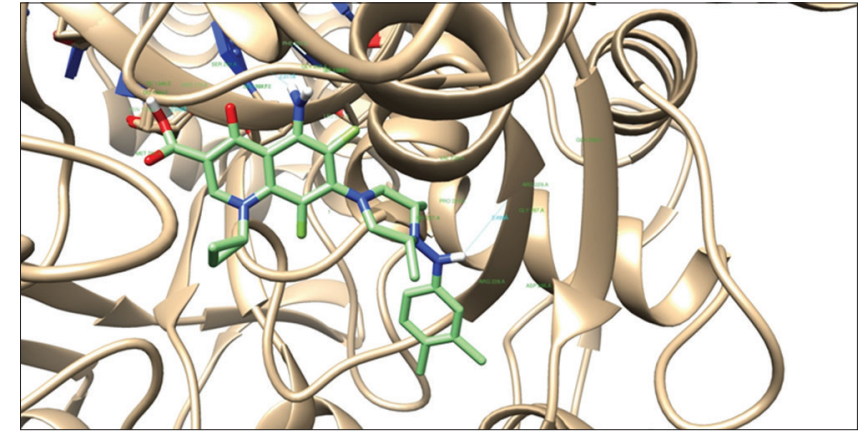

Fig. 3: H-bonds interactions between compound $\left(Q_{5}\right)$ with topoisomerase-II DNA gyrase enzyme of Gram-positive Staphylococcus aureus bacteria (5IWM)

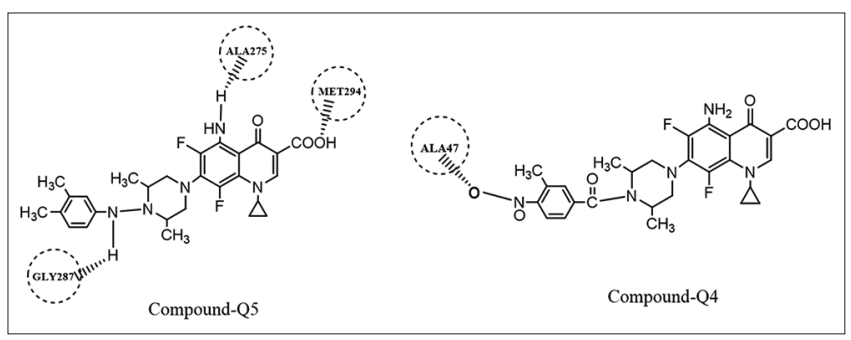

Fig. 4: Two-dimensional ligand interaction diagram of compounds $\mathbf{Q}_{5}$ with DNA gyrase and $\mathbf{Q}_{4}$ with Escherichia coli, the amino acid residues at the binding site are tagged in circles

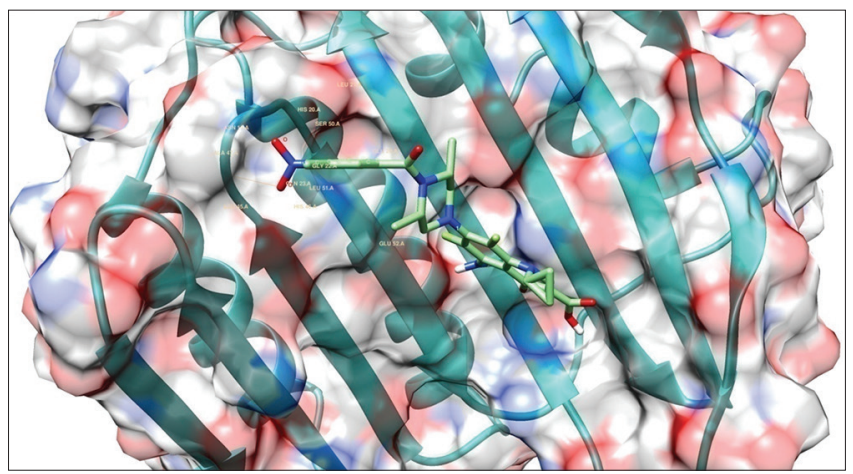

Fig. 5: H-bonds interactions between compound $\left(Q_{4}\right)$ with topoisomerase-IV enzyme of Gram-negative Escherichia coli bacteria (3FV5)

\section{Docking study}

Molecular docking studies were employed for the analysis with a training set composed of our synthesized compounds whose inhibitory activity is unknown. To find out the molecular facilities responsible for biological activity molecular docking studies was performed. From the docking studies, we predicted that all the synthesized compounds $\left(Q_{1}-Q_{10}\right)$ possess better antibacterial activity than the standard drugs (ciprofloxacin and sparfloxacin). By having good binding affinity with target protein, it could be used as a potential drug as antibacterial.

\section{Gram-positive bacteria docking studies}

Among all the docked compounds, $\mathrm{Q}_{5}, \mathrm{Q}_{6}, \mathrm{Q}_{4}$, and $\mathrm{Q}_{3}$ show good binding affinity and interaction with topoisomerase-II DNA gyrase enzyme (5IWM) with reference to standard drugs ciprofloxacin and sparfloxacin.

The interactions of $\mathrm{H}$ bonds with ligands and bacterial enzymes explained that Compound $Q_{5}$ at $3^{\text {rd }}$ position carboxylic oxygen forms hydrogen bond with MET294, $5^{\text {th }}$ position amino functional group forms

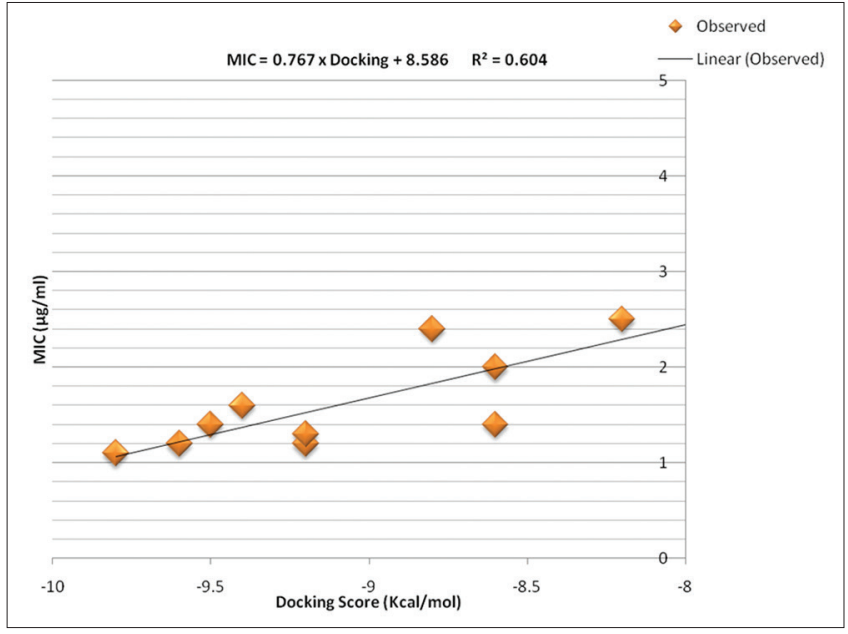

Fig. 6: Gram-positive (Staphylococcus aureus) correlation plot between minimum inhibitory concentrations $(\mu \mathrm{g} / \mathrm{ml})$ and docking scores $(\mathrm{Kcal} / \mathrm{mol})$

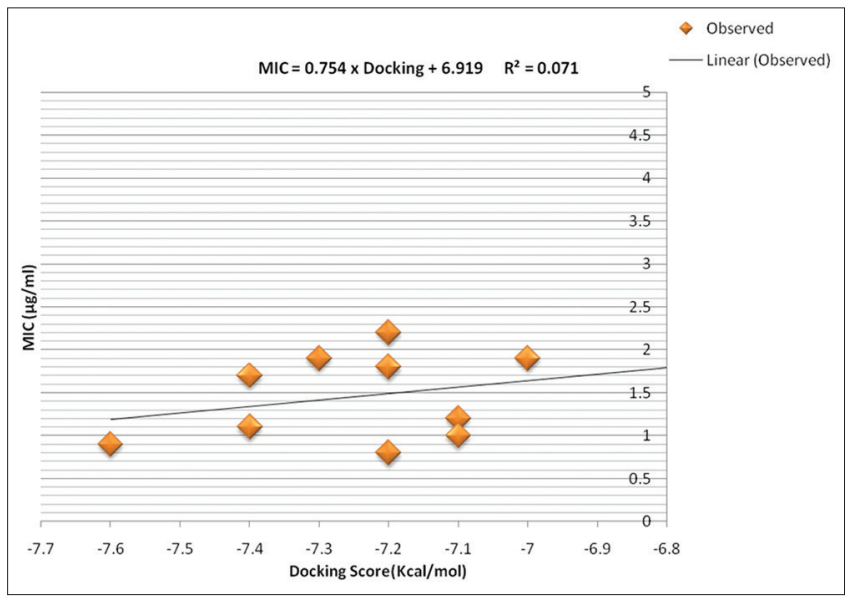

Fig. 7 Gram-negative (Escherichia coli) correlation plot between minimum inhibitory concentrations $(\mu \mathrm{g} / \mathrm{ml})$ and docking scores (Kcal/mol)

hydrogen bond with ALA275, $7^{\text {th }}$ position piperzinyl ring attachment dimethyl phenylamino group, and one of the methyl group hydrogen forms hydrogen bond with GLY287 as shown in Fig. 3 and interactions are shown in Fig. 4.

Compound $Q_{5}$ having higher dock score (-9.8) toward bacterial S. aureus enzyme than the standard ciprofloxacin $(-7.8)$ and sparfloxacin $(-8.2)$ drugs. We may declare that the higher docking score is due addition of dimethyl phenylamino group at the $7^{\text {th }}$ position of sparfloxacin structure. The remaining compounds docking score and hydrogen bond interactions are described in Table 2.

\section{Gram-negative bacteria docking studies}

Among all the docked compounds, $Q_{4^{\prime}}, Q_{g^{\prime}}, Q_{9}$, and $Q_{10}$ show good binding affinity and interaction with topoisomerase-IV enzyme (3FV5) with reference to standard drugs ciprofloxacin and sparfloxacin.

Compound $\mathrm{Q}_{4}$ at $7^{\text {th }}$ position piperzinyl ring attachment nitromethyl benzoyl oxygen forms hydrogen bond with ALA47 as shown in Fig. 5 and interactions are shown in Fig. 4.

Compound $\mathrm{Q}_{4}$ is having higher affinity $(-7.6)$ toward E. coli enzyme than the standard ciprofloxacin $(-7.3)$ and sparfloxacin $(-7.2)$ drugs. We may declare that the higher docking score is due addition 
of nitromethyl benzoyl group at the $7^{\text {th }}$ position of sparfloxacin structure. The remaining compounds docking score and hydrogen bond interactions are described in Table 2. The correlation between experimental data (MIC) and docking score of $S$. aureus and E. coli is displayed $0.604 \mathrm{r}^{2}$ and $0.071 \mathrm{r}^{2}$ (Figs. 6 and 7) which suggests that parameters for docking simulation are good for $S$. aureus and mild for E. coli in reproducing experimental orientation of synthesized compounds.

\section{CONCLUSION}

We have synthesized and characterized 10 new derivatives of sparfloxacin. All the molecules were studied for their interactions with topoisomerase-II DNA gyrase and topoisomerase-IV enzymes by molecular docking protocol. Among the tested molecules, Compounds $Q_{5}, Q_{6}, Q_{4}$, and $Q_{3}$ exhibited good docking score for Gram-positive bacteria and Compounds $Q_{4}, Q_{8}, Q_{9}$, and $Q_{10}$ for Gram-negative bacteria. In vitro, antibacterial activity of tested compounds shows mild activity against microorganisms used. In particular, Compounds $Q_{5} Q_{6}, Q_{4}$, and $Q_{3}$ possess significant Gram-positive activity and Compounds $Q_{4}, Q_{8}, Q_{9}$, and $Q_{10}$ possess significant Gram-negative activity. The results of antibacterial activity are supported by docking analysis only for $S$. aureus.

\section{AUTHOR'S CONTRIBUTION}

Hemanth K Sudheer Kumar make contributions to the conception, design and implementation of the research, to the analysis of the results and to the writing of the manuscript. Parameshwar $\mathrm{H}$ helped to supervise the project and gave final approval of the written manuscript.

\section{CONFLICTS OF INTEREST}

The author declares that they have no conflicts of interest.

\section{REFERENCES}

1. Hooper DC, Wolfson JS. The fluoroquinolones: Pharmacology, clinical uses, and toxicities in humans. Antimicrob Agents Chemother 1985;28:716-21.

2. Ball P. Bacterial resistance to fluoroquinolones: Lessons to be learned. Infection 1994;22:140-7.

3. Ball PL, Mandell YN, Tilloston G. Comparative tolerability of the newer fluoroquinolone antibacterials. Drug Saf 1999;21:407.

4. Ball PJ. Quinolone-induced QT interval prolongation: A not-sounexpected class effect. J Antimicrob Chemother 2000;45:557-9.

5. Anderson MI, Mac Gowan AP. Development of the quinolones. J Antimicrob Chemother 2003;51 Suppl S1:1-11
6. Koga H, Itoh A, Murayama S, Suzue S, Irikura T. Structure-activity relationships of antibacterial 6,7- and 7,8-disubstituted 1-alkyl1,4-dihydro-4-oxoquinoline-3-carboxylic acids. J Med Chem 1980;23:1358-63.

7. Pradip K, Ashish K, Susanta K, Swayansiddha T. Synthesis, biological evaluation, and docking studies of ciprofloxacin derivatives. Asian J Pharm Clin Res 2015;8:99-105.

8. Foroumadi A, Emami S, Hassanzadeh A, Rajaee M, Sokhanvar K, Moshafi MH, et al. Synthesis and antibacterial activity of N-(5benzylthio-1,3,4-thiadiazol-2-yl) and N-(5-benzylsulfonyl-1,3,4thiadiazol-2-yl)piperazinyl quinolone derivatives. Bioorg Med Chem Lett 2005; 15:4488-92.

9. Domagala JM, Hanna LD, Heifetz CL, Hutt MP, Mich TF, Sanchez JP, et al. New structure-activity relationships of the quinolone antibacterials using the target enzyme. The development and application of a DNA gyrase assay. J Med Chem 1986;29:394-404.

10. Elsea SH, Osheroff N, Nitiss JL. Cytotoxicity of quinolones toward eukaryotic cells. Identification of topoisomerase II as the primary cellular target for the quinolone CP-115,953 in yeast. J Biol Chem 1992;267:13150-3.

11. Chin NX, Gu JW, Yu KW, Zhang YK, Neu HC. In vitro activity of sparfloxacin. Antimicrob Agent Chemothe 1991;35:567-71.

12. Pasty BM. Clinical trial design and selected drug safety issues for antibiotics used to treat community-acquired pneumonia. Clin infect Dis Dec 2008;47:s176-9.

13. Shimada J, Nogita T, Ishibashi Y. Clinical pharmacokinetics of sparfloxacin. Clin Pharmacokinet 1993;25:358-69.

14. Nogita T, Ishibashi $Y$. The penetration of sparfloxacin into human plasma and skin tissues. J Antimicrob Chemother 1991;28:313-4.

15. Wise R, Honeybourne D. A review of the penetration of sparfloxacin into the lower respiratory tract and sinuses. J Antimicrob Chemother 1996;37 Suppl A:57-63.

16. Rubinstein E. Safety profile of sparfloxacin in the treatment of respiratory tract infections. J Antimicrob Chemother 1996;37 Suppl A:145-60.

17. Goa KL, Bryson HM, Markham A. Sparfloxacin. A review of its antibacterial activity, pharmacokinetic properties, clinical efficacy and tolerability in lower respiratory tract infections. Drugs 1997;53:700-25.

18. Lengauer T, Rarey M. Computational methods for biomolecular docking. Curr Opin Struct Biol 1996;6:402-6.

19. Ghalia S, Thanaa M. An In silico study of novel fluoroquinolones as inhibitors of DNA gyrase of Staphylococcus aureus. Int J Pharm Sci 2015;8:67-75

20. Gillespie SH. Medical Microbiology Illustrated. United Kingdom: Butterworth Heinemann Ltd.; 1994. p. 234-47.

21. Hawkey PM, Lewis DA. Medical Bacteriology a Practical Approach. United Kingdom: Oxford University Press; 1994. p. 181-94. 